\title{
The development of an oral GLP-I receptor agonist for the management of type 2 diabetes: evidence to date
}

This article was published in the following Dove Press journal:

Drug Design, Development and Therapy

\author{
Christina Antzal,* \\ Krishnarajah \\ Nirantharakumar ${ }^{2, *}$ \\ loannis Doundoulakis ${ }^{3}$ \\ Abd A Tahrani ${ }^{4-6}$ \\ Konstantinos A Toulis ${ }^{2,3}$ \\ 13rd Department of Internal Medicine, \\ Papageorgiou Hospital, Aristotle \\ University, Thessaloniki, Greece; \\ ${ }^{2}$ Institute of Applied Health Research, \\ University of Birmingham, Birmingham, \\ UK; ${ }^{3}$ Department of Endocrinology and \\ Diabetes, 424 General Military Hospital, \\ Thessaloniki, Greece; ${ }^{4}$ Institute of \\ Metabolism and Systems Research, \\ University of Birmingham, Birmingham, \\ UK; ${ }^{5}$ Centre for Endocrinology, Diabetes \\ and Metabolism, Birmingham Health \\ Partners, Birmingham, UK; ${ }^{6}$ Department \\ of Diabetes and Endocrinology, \\ Birmingham Heartlands Hospital, \\ Birmingham, UK \\ *These authors contributed equally to \\ this work
}

\begin{abstract}
Glucagon-like peptide 1 receptor agonists (GLP1-RA) are prominent agents in the therapeutics of type 2 diabetes mellitus due to their exemplary efficacy in both preprandial and postprandial glycemia, their safety, low risk of hypoglycemia, their multilevel pathophysiological superiority, weight loss and importantly the observed benefits in cardiovascular disease reduction. Their major drawback is the subcutaneous route of administration, constituting a barrier to adoption and reason for treatment discontinuation. Thus, the development of an oral GLP1-RA agent would promote medication adherence and quality of life, further consolidating its beneficial effects in real-life clinical practice. However, this task is hampered by suboptimal gastrointestinal protein absorption. Yet, the introduction of oral semaglutide, a modified form of semaglutide with the addition of a carrier sodium N-(8-[2hydroxybenzoyl] amino) caprylate, may have provided a safe and effective way to reach systemic circulation while other molecules are in development. Whether this molecule still has the impressive cardiovascular effects demonstrated with the use of its precursor remains to be explored. However, to date, its efficacy and safety have already been showcased in a randomized trial. More research is warranted in order to further consolidate these findings across different type 2 diabetes mellitus (T2DM) subpopulations, and adequately powered studies with a longer follow-up that would allow the exploration of microvascular and macrovascular complications are needed. Finally, studies comparing oral semaglutide and similar molecules with other currently established antidiabetic agents to evaluate the relative efficacy, the cost-effectiveness and further understand its place in T2DM therapeutic algorithm are needed. This review focuses on the development of oral GLP1-RA agents and summarizes the challenges, milestones and expected benefits associated with a successful introduction.
\end{abstract}

Keywords: glucagon-like peptide, pharmacokinetics, therapeutics

\section{Introduction}

Glucagon-like peptide 1 receptor agonists (GLP1-RA) constitute a class of antidiabetic medications with unique a set of characteristics. ${ }^{1}$ Despite within class variations in terms of clinical efficacy due to different biochemical structures and pharmacokinetics profiles, all members of GLP1-RA class (liraglutide, albiglutide, dulaglutide, lixisenatide and semaglutide) have shown signficant hypoglycaemic efficacy. Second, their safety profile is overall satisfactory considering the low risk of serious adverse events. Moreover, their supplementary effects on satiety and weight loss are essential in the management of type 2 diabetes mellitus (T2DM) to the extent that
Correspondence: Konstantinos A Toulis Department of Endocrinology, Diabetes and Metabolism, 424 General Military Hospital, Thessaloniki, Greece Tel +3023I 0381902

Email info@toulis.gr 
liraglutide has already been repurposed as an anti-obesity medication, and the $3 \mathrm{mg}$ dose is already approved and used in clinical practice. Last but not least, their favorable cardiovascular (CV) properties in blood pressure, endothelial function and myocardial metabolism ${ }^{2,3}$ exemplified in the LEADER trial ${ }^{4}$ and other studies, ${ }^{5}$ in which a significantly lower risk of CVD events was demonstrated with liraglutide in patients with T2DM, are of major clinical importance when considering the increased risk of $\mathrm{CV}$ and all-cause mortality in patients with T2DM. ${ }^{6}$

On top of the above hard clinical outcomes, the use of GLP1-RA is associated with unique beneficial effects from the pathophysiological perspective as well. This class has a glucose-dependent nature of action and is admittedly one of the most efficacious amongst glucose-lowering agents in addressing GLP-1 resistance and hyperglucagonemia, thus combating in multiple ways the multifactorial components of T2DM. ${ }^{1}$

Considering the above, it comes as no surprise that several formulations of GLP1-RA have been tested and developed and several members of the family have been introduced in everyday practice with different pharmacokinetic properties. In 2005, US Food and Drug Administration (FDA) approved exenatide for the treatment of type $2 \mathrm{DM}^{7}$ Long-acting liraglutide was approved in 2009 and 2010 by the European Medicines Agency and FDA, respectively, followed by lixisenatide (2013 EMA, 2016 FDA), albiglutide (2014 for both EMA and FDA) and dulaglutide (2014 for both EMA and FDA) ${ }^{8}$ On December 5, 2017, semaglutide was approved in the USA for its use as subcutaneous injection, while in Europe, semaglutide was approved by the EMA on February 8, 2018. ${ }^{9,10}$

However, notwithstanding the above admittedly wellgrounded benefits associated with the use of GLP1-RA, both the adoption and adherence to treatments may be considered as low ${ }^{11}$ in the real world. One of the major reasons is the high dropout rate, ${ }^{11}$ possibly due to the nausea-related side effects and importantly the fact that these formulations are till date injectable. The recent introduction of weekly instead of daily injection schemes is an advance, but still, the injectable therapy places a significant psychological and practical burden to patients, who may prioritize ease of use associated with an oral formulation over superior pathophysiological properties. On top of this, oral formulations do not require specific conditions of sterility and other precautions against possible particle contamination, which may also have cost implications. ${ }^{12}$
The above issues should be addressed by the development and introduction of oral GLP1-RA formulations, which are preferable by patients and related to better compliance and convenience. ${ }^{13,14}$ This is why the recent advances with semaglutide, the first and more advanced form of oral GLP1-RA, are considered a major step forward in the therapeutics of diabetes. ${ }^{1}$ These steps will be summarized in this review.

\section{Methods}

A systematic search using Medline and the Cochrane Library from inception to 29 September 2018 was performed to identify studies that semaglutide was used as a treatment for T2DM. We used keywords and search terms that had been identified from initial scoping searches, target references and browsing of database thesauruses (data supplementary 1). A basic search strategy was developed for PubMed and modified accordingly for other research engines. We also searched clinicaltrials.gov. References of relevant studies were perused. The study selection procedure is provided in Figure 1. A complementary literature search focusing on other similar agents was also undertaken.

\section{Results and discussion Synopsis of the precursor molecule (subcutaneous semaglutide)}

The injectable formulation of semaglutide has an increased affinity for native human GLP1. Semaglutide molecule resembles native GLP1 sharing a 94\% structural homology and is modified at 3 positions; position 8 , where alanine is substituted by alpha-aminoisobutyric acid and thus increasing resistance to DPP-4 degradation, position 34, where lysine is changed by arginine and thus, preventing wrong binding of C-18 fatty acid, and position 26 , where lysine is a acylated with a glutamate spacer ${ }^{15}$ to provide specific binding to albumin. The semaglutide molecule differs from its precursor molecule liraglutide in two positions; alanine at the second position is replaced by $\alpha$-aminoisobutyric acid and lysine 26 residue is acylated with a stearic diacid instead of palmitate. These changes result in extending semaglutide half-life to approximately 1 week, thus permitting a weekly delivery dosage scheme. ${ }^{16-18}$ The strong affinity for albumin also contributes to the long duration of action. ${ }^{17,19}$ The spacer region (glutamic acid moiety) between the $\mathrm{C}-18$ fatty di-acid sidechain and the peptide is considered to be important for potency, whereas the fatty acid is considered to be important for both potency and protraction. ${ }^{20}$ 


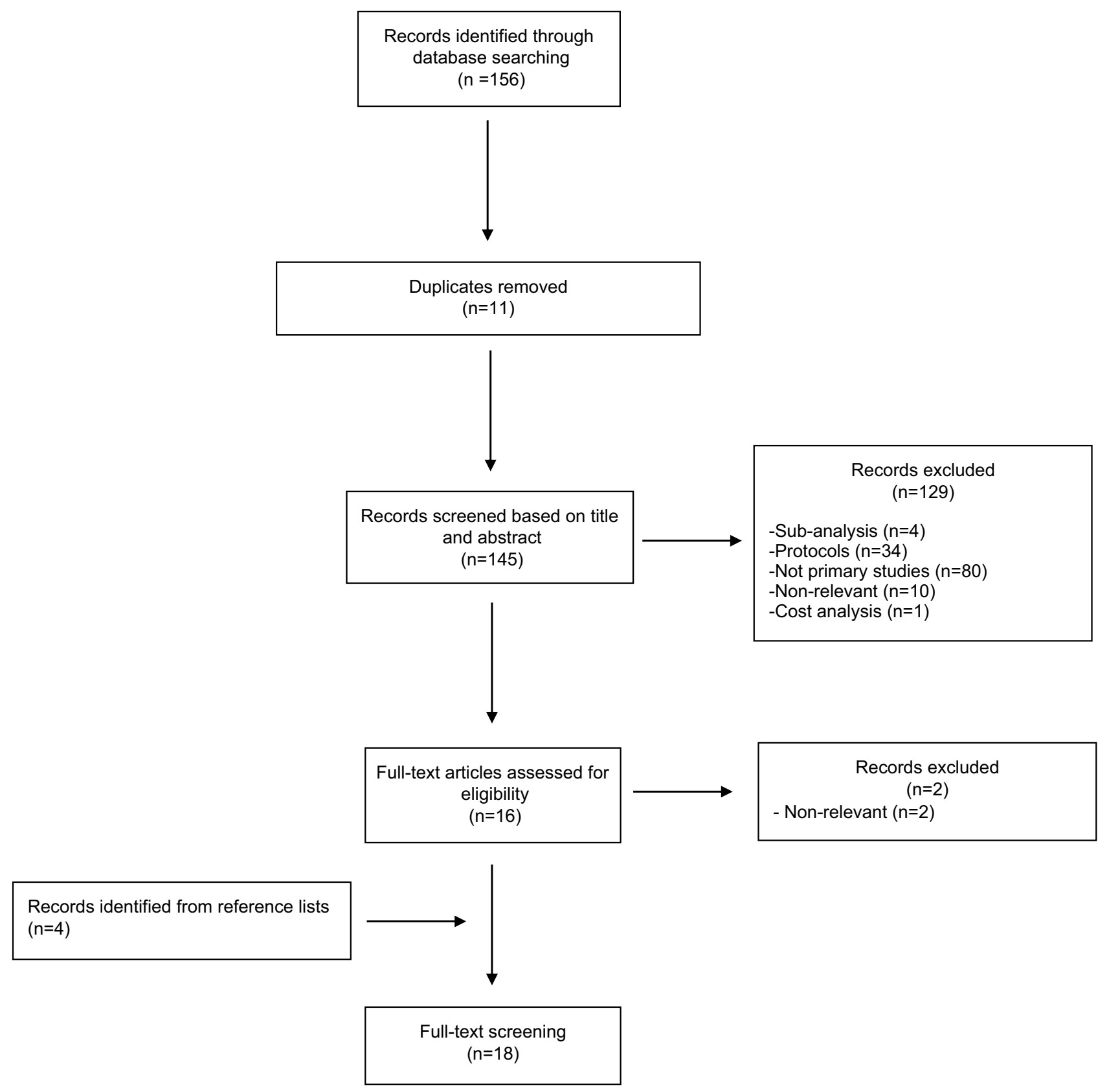

Figure I Summary of evidence search and selection.

The metabolism of semaglutide takes place in two steps: the first step is the proteolytic cleavage of the peptide backbone and the second one is $\beta$-oxidation (involving dehydrogenation, hydratation, dehydrogenation, thiolytic cleavage) of the fatty di-acid chain, procedures that are not confined to specific organs. ${ }^{15}$ Following $\beta$-oxidation, semaglutide degradation products are excreted mainly via urine and to lesser extent via the feces, involving hepatic metabolism in part. Amongst semaglutide metabolites, the most dominant metabolite is traceable up to $7.7 \%$ of all semaglutide material and found to consist of several co-eluting components. ${ }^{15}$

Moreover, the pharmacokinetics of semaglutide are not significantly affected by renal or hepatic impairment, ${ }^{21,22}$ and thus, no dose adjustment is required in these populations. Of note, the kidney function appears to be unaffected by the use of semaglutide. ${ }^{23}$ The level of creatine clearance also does not appear to affect the pharmacokinetics of semaglutide, ${ }^{22}$ yet clinical experience in patients with end-stage renal disease is limited and thus not recommended. No significant drug interactions were detected 
when semaglutide was coadministered with metformin, warfarin, digoxin, atorvastatin, ethinyl estradiol and levonorgestrel in healthy adults. ${ }^{18,24}$

The efficacy and safety of injectable semaglutide was explored in the SUSTAIN trials program. Overall, semaglutide was associated with improved glycaemic control, when used either as monotherapy or as add-on therapy in patients with T2DM against both placebo and active comparators (including other GLP-1 RAs). The results of the published SUSTAIN trials are summarized in Table 1. Compared with placebo, a significant decrease in HbAlc with both dosages $(1.45 \%$ and $1.55 \%$ for $0.5 \mathrm{mg}$ and 1.0 $\mathrm{mg}$, respectively) was observed in SUSTAIN 1. Moreover, body weight reduced significantly by $3.73 \mathrm{~kg}$ and $4.53 \mathrm{~kg}$ with $0.5 \mathrm{mg}$ and $1.0 \mathrm{mg}$, respectively. ${ }^{25}$ The magnitude of this effect is considered exceptionally high.

Adult patients with T2DM treated by metformin, thiazolidinediones/rosiglitazone or both without adequate glycaemic control after 3 months were enrolled in SUSTAIN 2 trial to explore the comparative effectiveness of semaglutide against sitagliptin. The primary outcome was the mean
HbA1c change from baseline to 56 weeks, which was significantly reduced in both semaglutide groups against sitagliptin. Notably, body weight reduced up to $\sim 6.0 \%$ for the highest dosage of semaglutide. ${ }^{26}$

SUSTAIN 3 trial compared semaglutide $1.0 \mathrm{mg}$ with exenatide extended release $2 \mathrm{mg}$ in adults with T2DM not controlled on one or two oral antidiabetic agents. After 56 weeks, Hb1Ac and the body weight were significantly decreased in the semaglutide group. ${ }^{27}$ The results were similar when semaglutide was compared to insulin glargine and dulaglutide, but importantly, fewer hypoglycaemic episodes were recorded with semaglutide. $^{28-30}$

In addition to its glucose- and weight-lowering efficacy, semaglutide, when used as add-on standard care in patients with T2DM and high cardiovascular disease, was found to be associated with significantly lower risk of cardiovascular death, nonfatal myocardial infarction, stroke and new or worsening nephropathy compared to placebo. ${ }^{31}$ This finding is regarded as of paramount importance in the long-term clinical care of patients with T2DM.

Table I Effectiveness of semaglutide versus control in $\mathrm{HbAlc}$ and weight reduction

\begin{tabular}{|c|c|c|c|c|c|c|}
\hline Study & Duration (weeks) & Route & Dose (mg) & Comparator & $\begin{array}{l}\text { HbAlc (\%) } \\
\text { Reduction }\end{array}$ & Weight (kg) Reduction \\
\hline SUSTAIN ${ }^{25}$ & 30 & subcutaneous & $\begin{array}{l}0.5 \\
1.0\end{array}$ & Placebo & $\begin{array}{l}-1.43^{*} \\
-1.53^{*}\end{array}$ & $\begin{array}{l}-2.75^{*} \\
-3.56^{*}\end{array}$ \\
\hline SUSTAIN $2^{27}$ & 56 & subcutaneous & $\begin{array}{l}0.5 \\
1.0\end{array}$ & Sitagliptin $100 \mathrm{mg}$ & $\begin{array}{l}-0.77^{*} \\
-1.06 *\end{array}$ & $\begin{array}{l}-2.35^{*} \\
-4.2^{*}\end{array}$ \\
\hline SUSTAIN $3^{28}$ & 56 & subcutaneous & 1.0 & Exanatide ER & $-0.62 *$ & $-3.78^{*}$ \\
\hline SUSTAIN $4^{29}$ & 30 & subcutaneous & $\begin{array}{l}0.5 \\
1.0 \\
\text { (+insulin) }\end{array}$ & Glargine U100 & $\begin{array}{l}-0.38^{*} \\
-0.8 I^{*}\end{array}$ & $\begin{array}{l}-4.62^{*} \\
-6.33^{*}\end{array}$ \\
\hline SUSTAIN $5^{31}$ & 30 & subcutaneous & $\begin{array}{l}0.5 \\
1.0 \\
\text { (+insulin) }\end{array}$ & Placebo & $\begin{array}{l}-1.35^{*} \\
-1.75^{*}\end{array}$ & $\begin{array}{l}-2.31^{*} \\
-5.06^{*}\end{array}$ \\
\hline SUSTAIN $6^{26}$ & 104 & subcutaneous & $\begin{array}{l}0.5 \\
1.0\end{array}$ & Placebo & $\begin{array}{l}-0.7^{*} \\
-1.0^{*}\end{array}$ & $\begin{array}{l}-2.9^{*} \\
-4.3^{*}\end{array}$ \\
\hline SUSTAIN $7^{30}$ & 40 & subcutaneous & $\begin{array}{l}0.5 \\
1.0\end{array}$ & $\begin{array}{l}\text { Delaglutide: } 0.75 \\
\text { I.5 }\end{array}$ & $\begin{array}{l}-0.4^{*} \\
-0.4 I^{*}\end{array}$ & $\begin{array}{l}-2.26^{*} \\
-3.55^{*}\end{array}$ \\
\hline Davies et al ${ }^{10}$ & 26 & Per Os & $\begin{array}{l}2.5 \\
5.0 \\
10.0 \\
20.0 \\
40.0\end{array}$ & Placebo & $\begin{array}{l}-0.4^{*} \\
-0.9^{*} \\
-1.2^{*} \\
-1.4^{*} \\
-1.6^{*}\end{array}$ & $\begin{array}{l}-0.9 \\
-1.5 \\
-3.6^{*} \\
-5.0^{*} \\
-5.7^{*}\end{array}$ \\
\hline
\end{tabular}

Notes: $* P<0.01$.

Abbreviations: SUSTAIN, semaglutide unabated sustainability in treatment of type 2 diabetes; s.c, subcutaneous; p.os, per os; HbAlc, glycated haemoglobin Alc. 
Similar to other GLP-1 RA, nausea and other gastrointestinal (GI)-related symptoms were the most common complications reported in the SUSTAIN trials. ${ }^{25}$ Nausea was reported up to a quarter of patients receiving semaglutide (placebo group 8\%). Diarrhea was less frequent; it was reported to $13 \%$ of patients receiving $0.5 \mathrm{mg}$ semaglutide and $11 \%$ of patients receiving $1 \mathrm{mg}$ semaglutide (placebo group 2\%). No case of pancreatitis or pancreatic or medullary cancer was documented, while cases of cholelithiasis were rare. ${ }^{25}$ In a large population consisting of 3297 patients (SUSTAIN 6 trial), the incidence of the above GI disorders was found to be similar. Of note, the rates of malignant neoplasms were similar in the pooled semaglutide groups $(0.5$ and $1.0 \mathrm{mg}$ ) compared to placebo, although the highest rate was noted in the semaglutide group receiving $1.0 \mathrm{mg} .{ }^{31}$ Microvascular outcomes related to diabetic retinopathy complications were found to be higher in semaglutide group compared to the placebo group, as expected in the context of rapid HbAlc reductions. These events were mostly mild or moderate and recorded mainly in patients with significant background diabetic retinopathy. ${ }^{12}$ This imbalance in retinopathy outcomes was absent in SUSTAIN 1-5 trials, yet not infrequently reported following abrupt glycaemic improvement (such as in Diabetes Control and Complications Trial and in newly diagnosed patients with T2D in the UK Prospective Diabetes Study (UKPDS 33), or in introduction of insulin in everyday clinical practice). On the other hand, the incidence of new or worsening nephropathy was significantly decreased in the semaglutide group compared to placebo. ${ }^{31}$

\section{Oral semaglutide}

\section{Challenges regarding the development of oral GLP-I RA}

The major barriers preventing the oral administration of a peptide or protein drug are presented by the enzymemediated peptide degradation and ensuing hydrolysis, the low permeability of intestinal epithelium and its mucus layer, and the $\mathrm{pH}$ differences between the "acidic" stomach and the basic intestinal lumen. ${ }^{13,14,32}$ These challenges could not be resolved by increasing the administered dose, since this can lead to a parallel increase in the incidence of side effects. Consequently, other strategies for oral administration have been tested and developed, including (but not limited to) coadministration with enzyme inhibitors, structural modifications [cyclization, PEGylation, fatty acid conjugates of polypeptides (lipidization), fusing therapeutic proteins to cobalamin (vitamin $\mathrm{B}_{12}$ )], and the use of delivery carriers or absorption enhancers. ${ }^{32}$

Novo Nordisk finally followed the latter approach (using absorption enhancers) for the development of oral semaglutide. The selected absorption enhancer was a system known as the Eligen ${ }^{\mathrm{TM}}$ oral drug delivery system, ${ }^{33}$ a platform based on the use of synthetic nonacylated amino acid as carriers, via the natural passive transcellular GI transport. Specifically, semaglutide was combined with sodium N-(8-[2-hydroxybenzoyl] amino) caprylate (SNAC), which is a small-chain fatty acid that facilitates semaglutide absorption by acting transcellularly on the gastric mucosa through a localized $\mathrm{pH}$ increase. ${ }^{34,35}$ Figure 2 visualizes how the Eligen System works in combination with semaglutide.

Considering its novelty, it is no wonder that in vivo evidence regarding SNAC safety and tolerability is still scarce. Riley et $\mathrm{al}^{36}$ evaluated the toxicity of SNAC in rats. In this study, Sprague-Dawley rats received SNAC alone at $2 \mathrm{~g} / \mathrm{kg}$ per day orally (for 10-13 weeks), resulting in severe toxicity and death. When administrated orally SNAC at levels of $0.1,0.5$ or $1 \mathrm{~g} / \mathrm{kg}$ per day to Wistar rats for 13 weeks, the results indicated that the maximum dose at which no toxic or adverse event was recorded (no-observed-adverse-effect level, NOAEL) was that of $1 \mathrm{~g} / \mathrm{kg}$ daily. ${ }^{36}$ Oral SNAC at $1 \mathrm{~g} / \mathrm{kg}$ per day was also administered to pregnant Sprague-Dawley rats, resulting in a minor decrease in maternal body weights, prolonged gestation and an increase in stillbirths. ${ }^{36}$ When 0.1 g SNAC was combined with vitamin B12 (cobala$\mathrm{min}$ ), the combination treatment provided a greater mean absolute bioavailability, compared to B12 alone and was shown to be well tolerated. ${ }^{37}$ The half-life of oral semaglutide was approximately 1 week, analogous to that of subcutaneous semaglutide, suggesting that the elimination phase of semaglutide administered orally is comparable with that observed with subcutaneous administration. Given a similar pattern of systemic exposure between the two routes of semaglutide administration, it is plausible to expect a similar pattern of efficacy and safety. Collectively, this evidence suggested that the use of SNAC as a method of overcoming absorption barriers should be a safe approach, considering that the dose under study is several times lower the NOAEL. However, evidence suggests that minor, reversible epithelial microdamage does occur with the use of SNAC and whether chronic, repeat exposure and accumulation of such microdamage may overcome the natural self-healing 


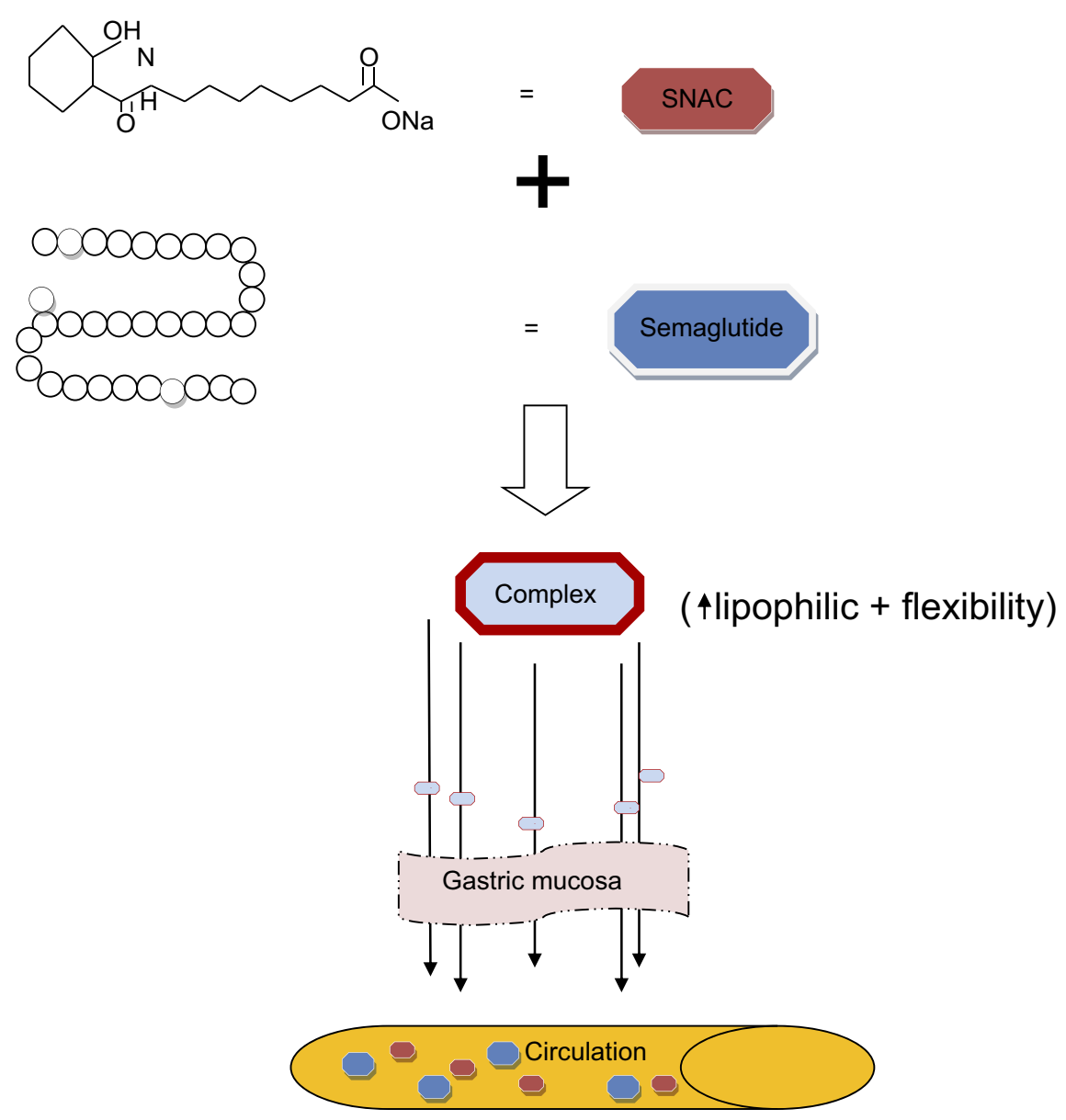

Figure 2 The Eligen System in the oral semaglutide formulation. Sodium N-(8-[2-hydroxybenzoyl] amino) caprylate (SNAC) is connected with semaglutide, providing a new complex with high lipophilic and flexible properties. This complex passes through the mouth to gastric mucosa, acting by increasing the $\mathrm{pH}$ and resulting to the semaglutide deliverance at the circulation.

capacity to restore the integrity of the barrier is naturally still a cause for concern.

\section{Oral semaglutide in specific subpopulations}

A crucial step in the development was to explore the safety of the molecule in patients with hepatic or renal impairment. Baekdal et al evaluated the pharmacokinetic effect of oral semaglutide (once-daily oral semaglutide $5 \mathrm{mg}$ for 5 days followed by $10 \mathrm{mg}$ for 5 days) in 56 patients with hepatic impairment. Patients were classified into four groups according to Child-Pugh criteria (from normal hepatic function to severe hepatic impairment). No significant difference in semaglutide plasma concentration across the four groups was observed and adverse events were similar across groups. ${ }^{38}$ In a study by Granhall et al, patients were categorized into five groups from normal renal function to end-stage renal disease on the basis of estimated creatinine clearance. The patients were administered the same dosage of oral semaglutide in gradually increasing steps $(5 \mathrm{mg}$ for 5 days followed by 10 $\mathrm{mg}$ for 5 days). Renal function was shown to have a neutral effect on the half-life of semaglutide, and similarly, hemodialysis did not affect the pharmacokinetics of this drug. ${ }^{39}$ Thus, oral semaglutide was generally considered to be safe for use in patients with liver and chronic kidney disease.

Since the absorption of oral semaglutide is influenced by the $\mathrm{pH}$ level, it was reasonable to explore whether the use of commonly prescribed proton pump inhibitors could change its pharmacokinetics. A single-center, randomized, open-label study in 54 healthy participants evaluated a possible interaction between omeprazole on its highest dosage $(40 \mathrm{mg}$ ) and oral semaglutide (5 mg for 5 days, followed by $10 \mathrm{mg}$ for 5 days). Using semaglutide area under the plasma concentration-time curve over $24 \mathrm{hrs}$ as the primary outcome, the main finding was a minor, yet not statistically significant increase in semaglutide 
exposure when the two drugs were co-prescribed. However, the small study population, open-label design and the lack of blindness are significant limitations that need to be considered when interpreting the data. ${ }^{40}$

\section{Oral semaglutide: safety and efficacy data from trials}

A Phase II, 26-week randomized multicenter, open-label clinical trial, in which oral semaglutide was compared with subcutaneous semaglutide and placebo, was designed and finally recruited 1106 participants. The design involved multiple dosing schemes of oral semaglutide, including once-daily dosages of $2.5 \mathrm{mg}, 5 \mathrm{mg}, 10 \mathrm{mg}, 20$ $\mathrm{mg}$ or $40 \mathrm{mg}$. Moreover, two additional 40-mg dosages were evaluated: the slow escalation extending over 8 weeks $(5 \mathrm{mg}$ as first dose for 8 weeks and doubling dose every 8 weeks until $40 \mathrm{mg}$ ) and the fast escalation of 2 weeks ( $5 \mathrm{mg}$ for the first 2 weeks and doubling dose every 2 weeks until $40 \mathrm{mg}$ ). The most common adverse events were reported from the GI system. In the standard-dose escalation of oral semaglutide, GI adverse events were similar to the subcutaneous semaglutide, yet lower compared to placebo. In the oral semaglutide group, the percentage of patients reported GI adverse events were approximately doubled in the case of fast escalation compared to the standard dose of $2.5 \mathrm{mg}$ and $5 \mathrm{mg}$. Generally, the severity of adverse events was higher in the high-dose oral semaglutide, compared to the subcutaneous or placebo group. This may explain the higher rates of treatment discontinuation in these cases. Surprisingly enough, episodes of hypoglycemia were less common in both the oral and subcutaneous semaglutide groups compared to the placebo, a finding that may constitute an advantage over other hypoglycaemic agents. ${ }^{41}$

With regard to its efficacy, oral semaglutide was found to be of comparable efficacy in reducing HbA1c with the subcutaneous form, associated with a reduction up to $1.9 \%$ in $\mathrm{HbA} 1 \mathrm{c}$ and $6.9 \mathrm{~kg}$ in body weight. ${ }^{41}$ The reduction of $\mathrm{HbA1c}$ and weight from the oral administration of semaglutide compared with placebo or other treatments is summarized in Table 2.

A Phase III, 26-week, randomized, double-blinded, placebo-controlled trial, the PIONEER 1, exploring the oral form of semaglutide at 3-mg, 7-mg and 14-mg doses in patients with T2DM is currently ongoing and it is soon expected to be concluded. Novo Nordisk announced a preliminary analysis from PIONEER 1 trial in February 2018; the oral semaglutide group achieved a $0.8 \%, 1.3 \%$ and $1.5 \%$

Table 2 Effectiveness of oral semaglutide versus control in glycemic control and weight reduction

\begin{tabular}{|c|c|c|c|c|c|}
\hline Study & $\begin{array}{l}\text { Duration } \\
\text { (weeks) }\end{array}$ & Semaglutide Dose (mg) & Comparator & $\begin{array}{l}\text { HbAlc (\%) } \\
\text { change }\end{array}$ & Weight change (kg) \\
\hline PIONEER I ${ }^{4 I}$ & 26 & $\begin{array}{l}3 \\
7 \\
14\end{array}$ & Placebo & $\begin{array}{l}-0.7^{*} \\
-1.2^{*} \\
-1.4^{*}\end{array}$ & $\begin{array}{l}-0.2 \\
-1.0^{*} \\
-2.6^{*}\end{array}$ \\
\hline PIONEER $2^{42}$ & 52 & 14 & Semaglutide $25 \mathrm{mg}$ & $-0.5^{*}$ & $-0.9 *$ \\
\hline PIONEER $3^{43}$ & 78 & $\begin{array}{l}3 \\
4 \\
17\end{array}$ & Sitagliptin $100 \mathrm{mg}$ & $\begin{array}{l}0.1 \\
-0.3^{*} \\
-0.7^{*}\end{array}$ & $\begin{array}{l}-0.8^{*} \\
-1.6^{*} \\
-2.4^{*}\end{array}$ \\
\hline PIONEER $4^{44}$ & 52 & 14 & $\begin{array}{l}\text { Placebo } \\
\text { Liraglutide } 1.8 \mathrm{mg}\end{array}$ & $\begin{array}{l}-1.4^{*} \\
-0.3^{*}\end{array}$ & $\begin{array}{l}-3.8^{*} \\
-1.9^{*}\end{array}$ \\
\hline PIONEER $5^{46}$ & 25 & 14 & Placebo & $-I^{*}$ & $-2.6 *$ \\
\hline PIONEER $7^{44}$ & 52 & On the basis of glycaemic control & Sitagliptin 100 mg & $N / A$ & $-2 . I^{*}$ \\
\hline Davies et $\mathrm{al}^{40}$ & 26 & $\begin{array}{l}2.5 \\
5.0 \\
10.0 \\
20.0 \\
40.0\end{array}$ & Placebo & $\begin{array}{l}-0.4^{*} \\
-0.9^{*} \\
-1.2^{*} \\
-1.4^{*} \\
-1.6^{*}\end{array}$ & $\begin{array}{l}-0.9 \\
-1.5 \\
-3.6^{*} \\
-5.0^{*} \\
-5.7^{*}\end{array}$ \\
\hline
\end{tabular}

Notes: *Denotes statistical significance at the level of 0.05 .

Abbreviation: HbAlc, glycated haemoglobin. 
reduction in $\mathrm{HbA} 1 \mathrm{c}$ and a weight loss of $1.7 \mathrm{~kg}, 2.5 \mathrm{~kg}$ and $4.1 \mathrm{~kg}$ at doses $3 \mathrm{mg}, 7 \mathrm{mg}$ and $14 \mathrm{mg}$, respectively. Further results and secondary end points are expected to be published after the completion of PIONEER 1 trial. $^{42}$

Following this announcement regarding the placebocontrolled PIONEER 1 trial, the main results from an active-controlled RCTs (trials in which oral semaglutide is compared to already approved antidiabetic medications) were also made public. These active comparators included SGLT2i empagliflozin (PIONEER 2), inhibitors of dipeptidyl peptidase 4 (DPP-4 inhibitor) sitagliptin (PIONEER 7) and GLP1-RA liraglutide (PIONEER 4).

In the 52-week PIONEER 2 trial, the main aim was to compare oral semaglutide with empagliflozin, in 421 patients inadequately controlled with metformin. Oral semaglutide was found to provide a significantly greater reduction in $\mathrm{HbA1c}$ and body weight compared to empagliflozin at 52 weeks. Fourteen milligrams of oral semaglutide resulted in a $1.3 \%$ improvement of $\mathrm{HbAlc}$ and $4.7 \mathrm{~kg}$ weight loss, while empagliflozin $25 \mathrm{mg}$ achieved a $0.8 \%$ decrease in $\mathrm{HbAlc}$ and $3.8 \mathrm{~kg}$ weight loss. ${ }^{43}$ The superiority of oral semaglutide in reducing HbAlc was observed as early as at week 26 . In the PIONEER 3 trial, similar results were reported showing the superiority of oral semaglutide at 78 weeks $(7 \mathrm{mg}$ and $14 \mathrm{mg}$ achieved: $0.7 \%$ and $1.1 \% \mathrm{HbA} 1 \mathrm{c}$ reduction, $2.7 \mathrm{~kg}$ and $3.5 \mathrm{~kg}$ weight loss) compared to the $100 \mathrm{mg}$ sitagliptin $(0.4 \% \mathrm{HbA} 1 \mathrm{c}$ reduction, $1.1 \mathrm{~kg}$ weight loss) in patients with T2DM inadequately controlled with metformin, with or without sulfonylurea. ${ }^{44}$ Analyses from PIONEER 4 and 7, both with 52-week duration, were also announced. In PIONEER 4, 14 mg oral semaglutide was compared with $1.8 \mathrm{mg}$ liraglutide ( $1.2 \%$ vs $0.9 \%$ $\mathrm{HbAlc}$ reduction, $5 \mathrm{~kg}$ vs $3.1 \mathrm{~kg}$ weight loss) and was found to provide comparable glycemic control and significant weight loss. In the open-label PIONEER 7, flexible once-daily adjustable dose of oral semaglutide ( $3 \mathrm{mg}, 7 \mathrm{mg}, 14 \mathrm{mg}$; $\mathrm{n}=253$ ) was compared to $100 \mathrm{mg}$ sitagliptin in patients whose type 2 diabetes was uncontrolled with one to two oral glucose-lowering medication. Flexible dose adjustment of oral semaglutide led to superior glycemic control and weight loss at week 52 versus sitagliptin and was well tolerated. Adverse events were more frequent in patients receiving oral semaglutide, yet generally mild and consistent with the GLP-1 receptor antagonist class (nausea). Discontinuation due to adverse events was more frequent for oral semaglutide (PIONEER 4: 11\%, PIONEER 7: $9 \%$ ) compared to liraglutide (9\%) and sitagliptin $(3 \%)$ users. ${ }^{45,46}$

Oral semaglutide was further investigated against placebo with regard to its cardiovascular safety (PIONEER 6) in patients considered to be at high cardiovascular risk, safety and efficacy in patients with moderate renal impairment (PIONEER 5), and safety and efficacy as an add-on therapy to insulin-treated patients (PIONEER 8). In PIONEER 6 trial, 3183 patients with T2DM were randomized to either oral semaglutide or placebo were observed for cardiovascular events up to a median of approximately 16 months. Major adverse cardiovascular events occurred in 61 of 1591 patients $(3.8 \%)$ in the oral semaglutide group and in 76 of $1592(4.8 \%)$ in the placebo group (hazard ratio, $0.79 ; 95 \% \mathrm{CI}, 0.57-1.11 ; p<0.001$ for noninferiority). ${ }^{47}$ Despite its relative short duration and low number of events, the cardiovascular risk profile of oral semaglutide appears to be (at least) not inferior to that of placebo. In a 26-week RCT (PIONEER 5), involving 163 patients randomized to oral semaglutide (14 $\mathrm{mg}$ once daily) and 161 patients to the placebo, oral semaglutide was shown to provide a superior glycemic control (estimated treatment difference of $0.8 \%$ ) and body weight loss (estimated treatment difference of $-2.5 \mathrm{~kg}$ ) than placebo and was found not to affect renal function. ${ }^{48}$ As an add-on to insulin with or without metformin (PIONEER 8), oral semaglutide (at doses of 3, 7 and $14 \mathrm{mg}$ ) was shown to be associated with superior HbAlc and body weight reductions versus placebo as early as at week 26 and achieved further reduction in insulin need (except dose $3 \mathrm{mg}$ ) at week 52, without significantly increasing the rate of hypoglycemia. ${ }^{49}$

The evolution of research starting from the first SUSTAIN trial to the creation of the new oral form of semaglutide $^{50-55}$ is depicted in Figure 3.

\section{Areas for future research}

The increasing incidence of T2DM, the need to reduce its burden and the better understanding of its pathogenesis have led to the discovery of new antidiabetic agents over the course of the last decade. Suboptimal results regarding the reduction in diabetes-related large-vessel disease and the recent encouraging cardiovascular disease (CVD) findings have refreshed the interest in the properties of antidiabetic agents, over and beyond the narrow perspective of HbAlc reduction. This is particularly stressed in the latest consensus report by the $\mathrm{ADA}$ and $\mathrm{EASD},{ }^{56}$ in which GLP-1 RAs are generally recommended as the first injectable therapy and the presence of heart disease, kidney disease and obesity are co-factored in the selection of antidiabetic agents. In this notion, the family of GLP1-RAs seems to concentrate on a unique set of 


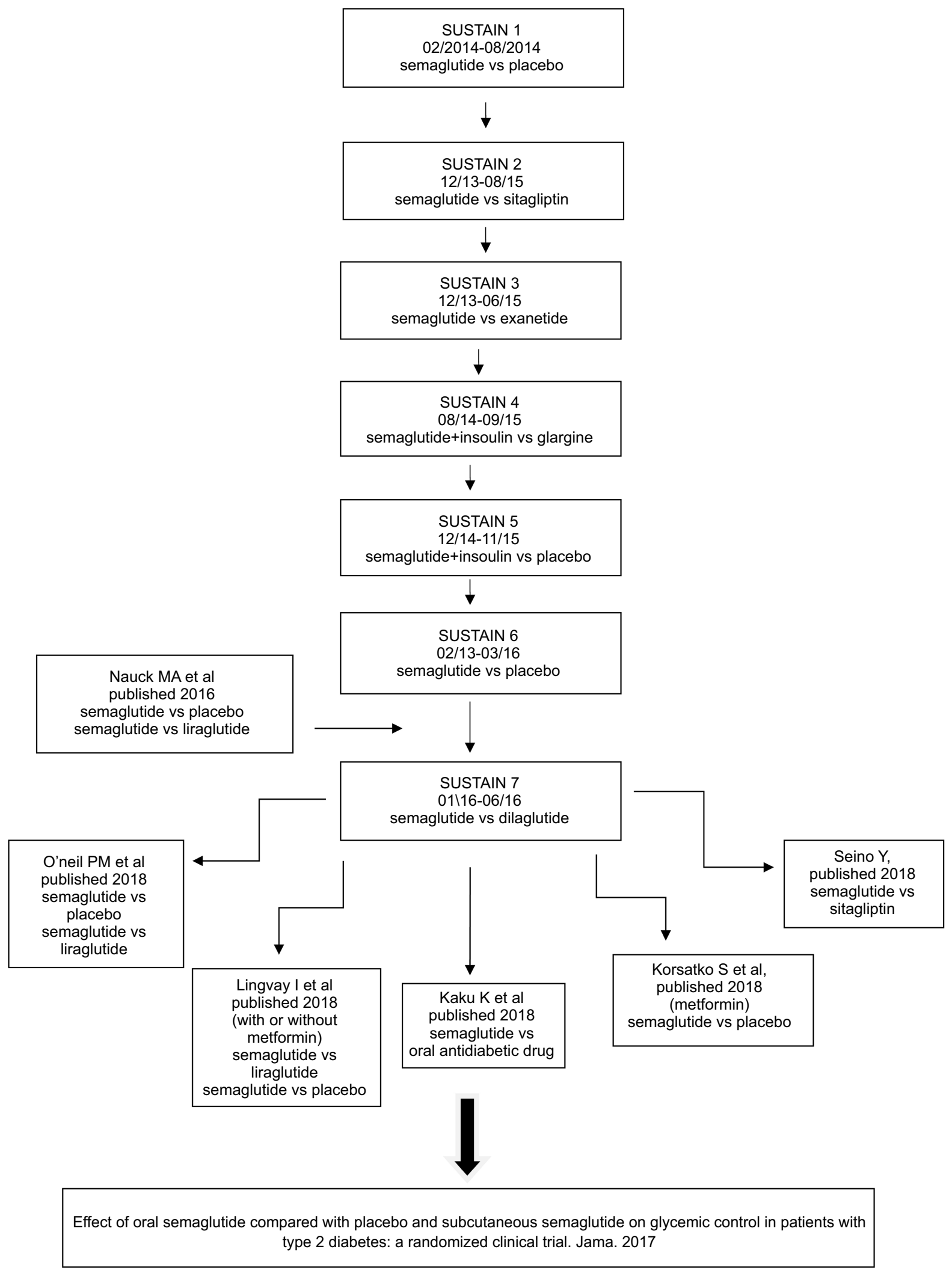

Figure 3 The steps from subcutaneous semaglutide to the creation of oral semaglutide. 
favorable characteristics and one major drawback. The favorable profile refers to their exemplary efficacy in addressing hyperglycemia, their safety, low risk of hypoglycemia, their multilevel pathophysiological superiority, weight loss and the reported benefits in CVD reduction. Their major drawback is the subcutaneous route of administration, constituting barrier to adoption and reason for treatment discontinuation.

Thus, any agent that would incorporate and maintain the above set of characteristics while addressing this major drawback would be a more than desirable addition to the class of GLP1-RAs, since it would promote medication adherence, quality of life and thus further consolidating its beneficial effect in real-life clinical practice. This task is not straightforward since several issues regarding the adequate protein absorption should be addressed before an oral GLP1-RA would reach systemic circulation in a safe and effective dose. Yet, this may be currently a fact with the introduction of oral semaglutide, a modified form of semaglutide with the addition of SNAC carrier.

On the basis of relevant evidence, this novel molecule seems to maintain the safety and efficacy of the subcutaneous form, along with the added benefits of weight loss and minimization of hypoglycemic episodes. Whether this molecule has still the impressive CVD effects is demonstrated with the use of its precursor remains to be explored. Till date though, its efficacy and safety has already been showcased in a randomized and parallel group trial, a study design associated with lower risk of bias and carry-over effects. The strengths of this trial also refer to its study size, the constitution of the study population involving patients from different countries, both of which contribute to the generalization of the findings. Moreover, several doses of oral semaglutide as well as the two types of treatment escalation were explored and results are provided for each of them. On the other hand, several shortcomings of the study should be reported to place the context for interpretation of its findings. First, this was an open-label, Phase II trial (lacks blinding), the duration of the study and followup were short, and thus, no data on hard outcomes, such as $\mathrm{CV}$ disease risk reduction and mortality, were analyzed. ${ }^{41}$

Overall, oral semaglutide, the single oral GLP1-RA agent, seems to be an effective and safe hypoglycemic agent in T2DM and might be considered as a promising future alternative option and an add-on therapy to further improve $\mathrm{Hb} 1 \mathrm{Ac}$ and reduce weight, while securing a low risk of hypoglycaemic episodes. More research with Phase III trials is warranted in order to further consolidate these findings across different T2DM subpopulations. Moreover, studies with a longer follow-up are needed for the evaluation of oral semaglutide with regard to microvascualr or macrovascular complications of DM, cardiovascular disease as well as mortality and morbidity. Studies comparing oral semaglutide with other currently established antidiabetic agents to evaluate the relative efficacy and costeffectiveness are eagerly needed. Finally, the development of similar molecules, permitting the safe and effective oral administration of GLP1-RA, would expand the therapeutic armamentarium of T2DM.

\section{Abbreviations list}

GLP1-RA, Glucagon-like peptide 1 receptor agonist; HbA1c, hemoglobin A1c; T2DM, type 2 diabetes mellitus; $\mathrm{CV}$, cardiovascular; FDA, food and drug administration; EMA, European Medicines Agency; SNAC, sodium N-(8[2-hydroxybenzoyl] amino) caprylate; GIP, gastric inhibitory polypeptide.

\section{Acknowledgments}

AAT is a clinician scientist supported by the National Institute for Health Research in the UK. The views expressed in this publication are those of the authors and not necessarily those of the National Health Service, the National Institute for Health Research, or the Department of Health (UK).

\section{Author contributions}

All authors contributed towards data analysis, drafting and critically revising the paper, gave final approval of the version to be published, and agreed to be accountable for all aspects of the work.

\section{Disclosure}

Dr Doundoulakis, Dr Antza, and Dr Toulis report no conflicts of interest in this work. Dr Nirantharakumar reports grants from National Institute for Health Research, MRC Medical Research Council, Diabetes UK, Vifor, and AstraZeneca, and personal fees from Merck Sharp \& Dohme, Sanofi, and Boehringer Ingelheim, outside of and unrelated to the submitted work. Dr Tahrani reports grants, personal fees, and non-financial support from NovoNordisk, personal fees and non-financial support from Eli Lilly, Boehringer Ingelheim and AstraZeneca, and personal fees from Janssen, during the conduct of the 
study. The authors report no other conflicts of interest in this work.

\section{References}

1. Tahrani AA, Barnett AH, Bailey CJ. Pharmacology and therapeutic implications of current drugs for type 2 diabetes mellitus. Nat Rev Endocrinol. 2016;12(10):566-592. doi:10.1038/nrendo.2016.86

2. Burgmaier M, Heinrich C, Marx N. Cardiovascular effects of GLP-1 and GLP-1-based therapies: implications for the cardiovascular continuum in diabetes? Diabetic Med. 2013;30(3):289-299. doi:10.1111/ j.1464-5491.2012.03746.x

3. Saraiva FK, Sposito AC. Cardiovascular effects of glucagon-like peptide 1 (GLP-1) receptor agonists. Cardiovasc Diabetol. 2014;13:142. doi:10.1186/1475-2840-13-80

4. Marso SP, Daniels GH, Brown-Frandsen K, et al. Liraglutide and cardiovascular outcomes in type 2 diabetes. $N$ Engl J Med. 2016;375 (4):311-22. doi:10.1056/NEJMoa1603827

5. Toulis KA, Hanif W, Saravanan P, et al. All-cause mortality in patients with diabetes under glucagon-like peptide-1 agonists: A population-based, open cohort study. Diabetes Metab. 2017;43 (3):211-216. doi:10.1016/j.diabet.2017.02.003

6. Taylor KS, Heneghan CJ, Farmer AJ, et al. All-cause and cardiovascular mortality in middle-aged people with type 2 diabetes compared with people without diabetes in a large U.K. primary care database. Diabetes Care. 2013;36(8):2366-2371. doi:10.2337/dc12-1513

7. Mullard A. Oral GLP1 analogue rounds Phase II corner. Nat Rev Drug Discov. 2015;14:227.

8. Andersen A, Lund A. Glucagon-like peptide 1 in health and disease. Nature Reviews Endocrinology. 2018;14(7):390-403.

9. OZEMPIC ${ }^{\circledR}$ (semaglutide) injection $0.5 \mathrm{mg} / 1 \mathrm{mg}$ [prescribing information]; Novo Nordisk; 2017. Available from: https://www.novo-pi.com/ ozempic.pdf. Accessed July 27, 2019.

10. EMA. Summary of the European public assessment report (EPAR) for Ozempic. 2018. Available from: http://www.ema.europa.eu/ema/index. jsp?curl=/pages/medicines/human/medicines/004174/human_med 002211.jsp\&mid=WC0b01ac058001d124. Accessed July 27, 2019.

11. Edelman SV, Polonsky WH. Type 2 diabetes in the real world: the elusive nature of glycemic control. Diabetes Care. 2017;40 (11):1425-1432. doi:10.2337/dc16-1974

12. Vilsboll T, Bain SC, Leiter LA, et al. Semaglutide, reduction in glycated haemoglobin and the risk of diabetic retinopathy. Diabetes Obes Metab. 2018;20(4):889-897. doi:10.1111/dom.13172

13. Chung SW, Hil-lal TA, Byun Y. Strategies for non-invasive delivery of biologics. J Drug Target. 2012;20(6):481-501. doi:10.3109/ 1061186X.2012.693499

14. Hamman JH, Enslin GM, Kotze AF. Oral delivery of peptide drugs: barriers and developments. BioDrugs. 2005;19(3):165-177. doi:10.2165/00063030-200519030-00003

15. Jensen L, Helleberg H, Roffel A, et al. Absorption, metabolism and excretion of the GLP-1 analogue semaglutide in humans and nonclinical species. Eur J Pharm Sci. 2017;104:31-41. doi:10.1016/j. ejps.2017.03.020

16. Lorenz M, Evers A, Wagner M. Recent progress and future options in the development of GLP-1 receptor agonists for the treatment of diabesity. Bioorg Med Chem Lett. 2013;23(14):4011-4018. doi:10.1016/j.bmcl.2013.05.022

17. Lau J, Bloch P, Schaffer L, et al. Discovery of the once-weekly glucagon-like peptide-1 (GLP-1) analogue semaglutide. J Med Chem. 2015;58(18):7370-7380. doi:10.1021/acs.jmedchem.5b00726

18. Kapitza C, Nosek L, Jensen L, Hartvig H, Jensen CB, Flint A. Semaglutide, a once-weekly human GLP-1 analog, does not reduce the bioavailability of the combined oral contraceptive, ethinylestradiol/levonorgestrel. J Clin Pharmacol. 2015;55(5):497-504. doi:10.1002/jcph.443
19. Hall S, Isaacs D, Clements JN. Pharmacokinetics and clinical implications of semaglutide: a new glucagon-like peptide (GLP)-1 receptor agonist. Clin Pharmacokinet. 2018;57:1529-1538. doi:10.1007/ s40262-018-0668-z

20. Madsen K, Knudsen LB, Agersoe H, et al. Structure-activity and protraction relationship of long-acting glucagon-like peptide-1 derivatives: importance of fatty acid length, polarity, and bulkiness. $J$ Med Chem. 2007;50(24):6126-6132. doi:10.1021/jm070861j

21. Jensen L, Kupcova V, Arold G, Pettersson J, Hjerpsted JB. Pharmacokinetics and tolerability of semaglutide in people with hepatic impairment. Diabetes Obes Metab. 2018;20(4):998-1005. doi:10.1111/dom.13186

22. Marbury TC, Flint A, Jacobsen JB, Derving Karsbol J, Lasseter K. Pharmacokinetics and tolerability of a single dose of semaglutide, a human glucagon-like peptide-1 analog, in subjects with and without renal impairment. Clin Pharmacokinet. 2017;56(11):1381-1390. doi:10.1007/s40262-017-0528-2

23. Bloomgarden Z. The kidney and cardiovascular outcome trials. $J$ Diabetes. 2018;10(2):88-89. doi:10.1111/1753-0407.12616

24. Hausner H, Derving Karsbol J, Holst AG, et al. Effect of semaglutide on the pharmacokinetics of metformin, warfarin, atorvastatin and digoxin in healthy subjects. Clin Pharmacokinet. 2017;56(11):13911401. doi:10.1007/s40262-017-0532-6

25. Sorli C, Harashima SI, Tsoukas GM, et al. Efficacy and safety of onceweekly semaglutide monotherapy versus placebo in patients with type 2 diabetes (SUSTAIN 1): a double-blind, randomised, placebo-controlled, parallel-group, multinational, multicentre phase 3a trial. Lancet Diabetes Endocrinol. 2017;5(4):251-260. doi:10.1016/S2213-8587(17)30013-X

26. Ahren B, Masmiquel L, Kumar H, et al. Efficacy and safety of onceweekly semaglutide versus once-daily sitagliptin as an add-on to metformin, thiazolidinediones, or both, in patients with type 2 diabetes (SUSTAIN 2): a 56-week, double-blind, phase 3a, randomised trial. Lancet Diabetes Endocrinol. 2017;5(5):341-354. doi:10.1016/S22138587(17)30092-X

27. Ahmann AJ, Capehorn M, Charpentier G, Dotta F, Henkel E, Lingvay I. Efficacy and safety of once-weekly semaglutide versus exenatide ER in subjects with type 2 diabetes (SUSTAIN 3): a 56week, open-label, randomized clinical trial. Diabetes Care. 2018;41 (2):258-266. doi: $10.2337 / \mathrm{dc} 17-0417$

28. Aroda VR, Bain SC, Cariou B, et al. Efficacy and safety of onceweekly semaglutide versus once-daily insulin glargine as add-on to metformin (with or without sulfonylureas) in insulin-naive patients with type 2 diabetes (SUSTAIN 4): a randomised, open-label, parallel-group, multicentre, multinational, phase 3a trial. Lancet Diabetes Endocrinol. 2017;5(5):355-366.

29. Pratley RE, Aroda VR, Lingvay I, et al. Semaglutide versus dulaglutide once weekly in patients with type 2 diabetes (SUSTAIN 7): a randomised, open-label, phase $3 \mathrm{~b}$ trial. Lancet Diabetes Endocrinol. 2018;6(4):275-286. doi:10.1016/S2213-8587(18)30024-X

30. Rodbard HW, Lingvay I, Reed J, et al. Semaglutide added to basal insulin in type 2 diabetes (SUSTAIN 5): a randomized, controlled trial. J Clin Endocrinol Metab. 2018;103(6):2291-2301. doi:10.1210/ jc. $2018-00070$

31. Marso SP, Bain SC, Consoli A, et al. Semaglutide and cardiovascular outcomes in patients with type 2 diabetes. $N$ Engl J Med. 2016;375 (19):1834-1844. doi:10.1056/NEJMoa1607141

32. Bruno BJ, Miller GD, Lim CS. Basics and recent advances in peptide and protein drug delivery. Ther Deliv. 2013;4(11):1443-1467. doi: $10.4155 /$ tde. 13.104

33. Ma S, We L, Yang H, Deng S, Anthony M, Jevnikar AM. Emerging technologies to achieve oral delivery of GLP-1 and GLP-1 analogs for treatment of type 2 diabetes mellitus (T2DM). Can J Biotech. 2017;1(1):1-10. doi:10.24870/cjb.2017-000107

34. Buckley STSS, Kirk RK, Knudsen LB. Mechanism of absorption mediated by SNAC in an oral formulation of semaglutide. Diabetes. 2017;66(suppl 61):1206-P. 
35. Goldberg M, Gomez-Orellana I. Challenges for the oral delivery of macromolecules. Nat Rev Drug Discov. 2003;2(4):289-295. doi: $10.1038 / \mathrm{nrd} 1067$

36. Riley MG, Castelli MC, Paehler EA. Subchronic oral toxicity of salcaprozate sodium (SNAC) in Sprague-Dawley and Wistar rats. Int J Toxicol. 2009;28(4):278-293. doi:10.1177/1091581809337737

37. Castelli MC, Wong DF, Friedman K, Riley MG. Pharmacokinetics of oral cyanocobalamin formulated with sodium N-[8-(2-hydroxybenzoyl)amino]caprylate (SNAC): an open-label, randomized, singledose, parallel-group study in healthy male subjects. Clin Ther. 2011;33(7):934-945. doi:10.1016/j.clinthera.2011.05.088

38. Baekdal TA, Thomsen M, Kupcova V, Hansen CW, Anderson TW. Pharmacokinetics, safety, and tolerability of oral semaglutide in subjects with hepatic impairment. J Clin Pharmacol. 2018;58:13141323. doi: $10.1002 /$ jcph.v58.10

39. Granhall C, Sondergaard FL, Thomsen M, Anderson TW. Pharmacokinetics, safety and tolerability of oral semaglutide in subjects with renal impairment. Clin Pharmacokinet. 2018;57:15711580. doi:10.1007/s40262-018-0649-2

40. Baekdal TA, Breitschaft A, Navarria A, Hansen CW. A randomized study investigating the effect of omeprazole on the pharmacokinetics of oral semaglutide. Expert Opin Drug Metab Toxicol. 2018;14 (8):869-877. doi:10.1080/17425255.2018.1488965

41. Davies M, Pieber TR, Hartoft-Nielsen ML, Hansen OKH, Jabbour S, Rosenstock J. Effect of oral semaglutide compared with placebo and subcutaneous semaglutide on glycemic control in patients with type 2 diabetes: a randomized clinical trial. JAMA. 2017;318(15):14601470. doi:10.1001/jama.2017.14752

42. Aroda VR, Rosenstock J, Terauchi Y, et al. PIONEER 1: randomized clinical trial comparing the efficacy and safety of oral semaglutide monotherapy with placebo in patients with type 2 diabetes. Diabetes Care. 2019:dc190749. doi:10.2337/dc19-0749

43. Montanya E, Rosenstock J, Canani LH, et al. 54-OR: oral semaglutide vs. empagliflozin added on to metformin monotherapy in uncontrolled type 2 diabetes: PIONEER 2. Diabetes. 2019;68(Supplement 1):54-OR. doi:10.2337/db19-54-OR

44. Rosenstock J, Allison D, Birkenfeld AL, et al. Effect of additional oral semaglutide vs sitagliptin on glycated hemoglobin in adults with type 2 diabetes uncontrolled with metformin alone or with sulfonylurea: the PIONEER 3 randomized clinical trial. JAMA. 2019;321:1466. doi:10.1001/jama.2019.2942

45. Pratley R, Amod A, Hoff ST, et al. Oral semaglutide versus subcutaneous liraglutide and placebo in type 2 diabetes (PIONEER 4): a randomised, double-blind, phase 3a trial. Lancet. 2019. doi:10.1016/ S0140-6736(19)31271-1

46. BUSE JB, BODE BW, CHO YM, et al. 983-P: effect and safety of flexible dose adjustment with oral semaglutide vs. sitagliptin in type 2 diabetes: PIONEER 7. Diabetes. 2019;68(Supplement 1):983-P. doi:10.2337/db19-983-P
47. Husain M, Birkenfeld AL, Donsmark M, et al. Oral semaglutide and cardiovascular outcomes in patients with type 2 diabetes. $N$ Engl $J$ Med. Epub June 11, 2019. doi:10.1056/NEJMoa1901118

48. Mosenzon O, Rosenlund S, Eriksson JW, et al. 1004-P: oral semaglutide vs. placebo in patients with type 2 diabetes and moderate renal impairment: PIONEER 5. Diabetes. 2019;68(Supplement 1):1004-P. doi:10.2337/db19-1004-P

49. ZINMAN B, ARODA VR, BUSE JB, et al. 985-P: oral semaglutide as add-on to insulin in T2D: PIONEER 8. Diabetes. 2019;68 (Supplement 1):985-P. doi:10.2337/db19-985-P

50. O'Neil PM, Birkenfeld AL, McGowan B, et al. Efficacy and safety of semaglutide compared with liraglutide and placebo for weight loss in patients with obesity: a randomised, double-blind, placebo and active controlled, dose-ranging, phase 2 trial. Lancet. 2018;392 (10148):637-649. doi:10.1016/S0140-6736(18)31773-2

51. Lingvay I, Desouza CV, Lalic KS, et al. A 26-week randomized controlled trial of semaglutide once daily versus liraglutide and placebo in patients with type 2 diabetes suboptimally controlled on diet and exercise with or without metformin. Diabetes Care. 2018;41 (9):1926-1937. doi:10.2337/dc17-2381

52. Korsatko S, Jensen L. Effect of once-weekly semaglutide on the counterregulatory response to hypoglycaemia in people with type 2 diabetes: a randomized, placebo-controlled, double-blind, crossover trial. Diabetes Obes Metab. 2018;20:2565-2573. doi:10.1111/ dom.2018.20.issue-11

53. Seino Y, Terauchi Y, Osonoi T, et al. Safety and efficacy of semaglutide once weekly vs sitagliptin once daily, both as monotherapy in Japanese people with type 2 diabetes. Diabetes Obes Metab. 2018;20 (2):378-388. doi:10.1111/dom.13082

54. Nauck MA, Petrie JR, Sesti G, et al. A phase 2, randomized, dosefinding study of the novel once-weekly human GLP-1 analog, semaglutide, compared with placebo and open-label liraglutide in patients with type 2 diabetes. Diabetes Care. 2016;39(2):231-241. doi:10.2337/dc15-0165

55. Kaku K, Yamada Y, Watada H, et al. Safety and efficacy of onceweekly semaglutide vs additional oral antidiabetic drugs in Japanese people with inadequately controlled type 2 diabetes: A randomized trial. Diabetes Obes Metab. 2018;20(5):1202-1212. doi:10.1111/ dom. 13218

56. Davies MJ, D'Alessio DA, Fradkin J, et al. Management of hyperglycaemia in type 2 diabetes, 2018. A consensus report by the American Diabetes Association (ADA) and the European Association for the Study of Diabetes (EASD). Diabetologia. 2018;61:2461-2498. doi:10.1007/s00125-018-4729-5

\section{Publish your work in this journal}

Drug Design, Development and Therapy is an international, peerreviewed open-access journal that spans the spectrum of drug design and development through to clinical applications. Clinical outcomes, patient safety, and programs for the development and effective, safe, and sustained use of medicines are a feature of the journal, which has also been accepted for indexing on PubMed Central. The manuscript management system is completely online and includes a very quick and fair peer-review system, which is all easy to use. Visit http://www. dovepress.com/testimonials.php to read real quotes from published authors. 